Revista Electrónica Complutense de Investigación en Educación Musical ISSN-e: 1698-7454

http://dx.doi.org/10.5209/RECIEM.53371

\title{
El conocimiento armónico como estrategia para mejorar la comprensión, la motivación y la creatividad en el aprendizaje del lenguaje musical
}

\author{
Elena Berrón Ruiz'; Inés María Monreal Guerrero²; Francisco José Balsera Gómez ${ }^{3}$
}

Recibido: 07 de diciembre de 2016 / Aceptado: 07 de julio de 2017

Resumen. En nuestro contexto socio-cultural occidental, la mayoría de la música que se produce y se consume está basada en el sistema tonal-armónico, que organiza el discurso musical a partir de la combinación de acordes con distintos grados de tensión y de reposo. En un intento por mejorar la formación musical de los alumnos y facilitarles la adquisición de los aprendizajes, se ha abordado la elaboración, puesta en práctica y evaluación de una serie de propuestas metodológicas para la iniciación a la educación musical desde un contexto tonal en la asignatura de Lenguaje Musical, dirigida a los alumnos de los dos primeros cursos de las enseñanzas elementales del Conservatorio Profesional de Música de Segovia. La investigación presentada en este artículo permite afirmar, como una de sus conclusiones fundamentales, que las actividades tonales desarrolladas en el aula han sido realmente útiles para ofrecer una educación integral, significativa y motivadora para los alumnos, al mismo tiempo que han potenciado su creatividad y les ha capacitado para la composición de canciones.

Palabras clave: sistema tonal-armónico; lenguaje Musical; creatividad; composición musical; conservatorio.

\section{[en] Harmonic knowledge as strategy to improve comprehension, motivation and creativity in ear training learning}

Abstract. In our Western sociocultural context, the vast majority of the music that it is produced and consumed is based on the tonal-harmonic system, which organises musical discourse thanks to the combination of chords with tension and release. In an attempt to improve the musical training of students and facilitate the acquisition of knowledge, different methodological proposals have been designed, implemented and evaluated from a tonal context in the Ear Training subject. Students of the two first courses in the elementary level at the Professional Conservatory of Music in Segovia have taken part in this study. The research included in this paper shows clearly that the suggested activities have been really useful to offer holistic, meaningful and motivating education to students, have fostered their creative abilities and have enabled them to write songs.

Keywords: Tonal-harmonic system; ear training; creativity; musical composition; conservatoire.

Sumario. 1. Introducción. 2. Definición del objeto de estudio. 3. Estudios previos relacionados con esta investigación. 4. Metodología de la investigación. 4.1. Método de investigación utilizado. 4.2.

1 Conservatorio Profesional de Música de Segovia

eberron@educa.jcyl.es

$2 \quad$ Universidad de Valladolid

ines.monreal@mpc.uva.es

3 Conservatorio Profesional de Música de Zaragoza

fbalsera75@gmail.com 
Selección de los participantes. 4.3. Técnicas e instrumentos para la recogida de datos. 5. Descripción de la intervención educativa para trabajar el sistema tonal-armónico. 5.1. Identificación auditiva de los acordes tonales. 5.2. Reconocimiento auditivo de intervalos. 5.3. Entonación memorística de escalas y arpegios. 5.4. Invención y adaptación de motivos rítmico-melódicos. 5.5. Aprendizaje, análisis y transcripción de canciones. 5.6. La principal aportación: la creación de canciones. 6. Análisis de los datos obtenidos. 6.1. Motivación hacia las actividades armónicas. 6.2. Utilidad del conocimiento armónico. 6.3. Dificultades encontradas. 7. Conclusiones. 8. Referencias bibliográficas.

Cómo citar: Berrón Ruiz, E.; Monreal Guerrero, I.M.; Balsera Gómez, F.J. (2017). El conocimiento armónico como estrategia para mejorar la comprensión, la motivación y la creatividad en el aprendizaje del lenguaje musical, en Revista Electrónica Complutense de Investigación en Educación Musical, 14, 305-327.

\section{Introducción}

En el campo de la pedagogía musical, los docentes no pueden olvidar que los niños que acuden a sus aulas ya saben mucha música. Por esta razón, el cometido del profesorado debe ser conseguir que los alumnos sean conscientes de los elementos constitutivos de la música a partir de sus conocimientos previos. En este sentido, Malbrán (1996) indica que las melodías "se presentan, retienen y analizan con el acompañamiento armónico y como discurso completo" (p. 68) y, en la misma línea, Furnó (2003) señala que los giros melódicos, las armonías y las cadencias de aquello que cantan y escuchan los niños están dejando unos sedimentos en su estructura cerebral, que son los que configuran las bases del lenguaje musical. Por ello, se considera que no tiene sentido empezar a explicarles cuestiones teóricas, como los nombres de las notas y su colocación en el pentagrama, si no pueden relacionarlo con lo que ya saben. En otras palabras, al igual que ocurre con el lenguaje verbal, los niños primero han de tener cierta comprensión intuitiva y práctica de la música que cantan y que escuchan y, cuando esto esté adquirido, ya vendrá el interés por conocer su escritura.

En nuestro contexto sociocultural, la música que se consume, se canta y se escucha a diario se rige por las normas del sistema tonal-armónico. Por este motivo, se parte de la convicción de que la base más sólida para llevar a cabo una buena educación musical debería proporcionarla dicha armonía, comenzando por los acordes tonales, generadores de tensión y de reposo.

Paradójicamente, en los planes de estudio de los conservatorios no se inician los estudios de armonía hasta el tercer curso de las enseñanzas profesionales, por lo que si los docentes no asumen la responsabilidad de tratar con anterioridad ciertos conceptos armónicos, tales como la formación de los acordes y su relación con la construcción de melodías, los alumnos adquirirán una visión parcial sobre la música que han estado estudiando durante los seis cursos anteriores. De ahí surge el interés por familiarizar a los alumnos con dichos elementos armónicos desde el principio de sus estudios musicales en el conservatorio. 


\section{Definición del objeto de estudio}

Ante la importancia de familiarizar a los alumnos con conceptos armónicos básicos que favorezcan el aprendizaje musical, en esta investigación se plantea, como eje vertebrador, valorar si el estudio sistemático de la música tonal entre el alumnado de conservatorio es más efectivo y eficaz cuando se trabajan la comprensión, la discriminación y el manejo de los acordes de tónica, subdominante y dominante desde el inicio de sus estudios musicales, utilizando para ello una metodología adaptada a sus capacidades y psicología infantil.

A lo largo del artículo, que resume parte de la investigación realizada para la elaboración de la Tesis Doctoral de Berrón (2016), se recoge cómo se han trabajado estos contenidos armónicos de forma integrada con el resto de los contenidos que se abordan tradicionalmente desde la asignatura de Lenguaje Musical. Tras su puesta en práctica en el aula, se han analizado los datos obtenidos, los cuales han permitido dar respuesta a los dos objetivos de investigación planteados, que son:

- Determinar la utilidad de iniciar a los alumnos en el conocimiento de las bases de la armonía tonal para mejorar su comprensión musical.

- Observar si las propuestas implementadas en el aula aumentan la motivación y creatividad del alumnado.

\section{Estudios previos relacionados con esta investigación}

Durante siglos, se ha pensado que el aprendizaje musical se basaba en el talento, de tal manera que un alumno servía o no para la música en función de sus aptitudes. No obstante, desde principios del siglo XX se empezaron a desarrollar distintas corrientes pedagógico-musicales de reconocidos autores, como Dalcroze (1980), Willems (1981), Kodály (1969), Orff y Keetman (1950), Suzuki (2007), Ward (1964) y Martenot (1993), que enfatizaban la importancia de una buena educación y del empleo de estrategias metodológicas adecuadas para que el aprendizaje de la música fuera accesible a todos los alumnos. Si se parte de la consideración de que el talento musical no es un rasgo innato capaz de transmitirse de forma hereditaria (Sloboda, 1985; Tafuri, 2006; Torres, 2011), aunque algunos tengan mayor facilidad que otros, es posible confiar en que una buena labor pedagógica podrá hacer de cualquier alumno una persona musicalmente educada.

En la música el aprendizaje es básicamente procedimental. Inicialmente no se aprenden contenidos, sino que se desarrollan destrezas (melódica, rítmica, armónica y memoria tonal) con un input declarativo, las cuales son previas a cualquier construcción de significado. Asimismo, se debe tener en cuenta que el conocimiento simbólico asociado al lenguaje de la música incluye una dimensión artística que confiere a las construcciones sonoras una significación que es añadida de forma cultural o personal (DeNora, 1986).

Investigadores como Hargreaves (1996) o Sloboda (1985) afirman que la cultura musical de los niños tiene que ver con la música de su entorno y han observado que el ambiente musical que les rodea influye en el desarrollo de sus destrezas musicales. En este sentido, Hargreaves (2002) afirma que "a los cinco años, los niños poseen un 
amplio repertorio de canciones tradicionales infantiles 'estándar' de su cultura, con las cuales pueden realizar tareas de reconocimiento y memoria mucho mejor que con el material musical no familiar" (p. 89).

En nuestro contexto cultural, Sierra (2008) señala que: "Hoy en día, las canciones comerciales difundidas por los medios de comunicación y recogidas en distintos formatos de audio y video constituyen las verdaderas 'canciones tradicionales infantiles estándar' de la cultura occidental" (p. 15), y estas canciones responden melódica y armónicamente a los tópicos tonales derivados de la tradición clásica europea: funciones, cadencias y sensaciones de final o de continuidad implícitas en la música. Contando de antemano con que los alumnos llegan al aula con estos conocimientos previos, y ya en el terreno de lo plenamente consciente, ¿cómo debería llevarse a cabo su educación musical?

En el estudio presentado en este artículo se parte de la conveniencia de basar la educación musical de los alumnos de los conservatorios españoles, desde los primeros niveles, en la música tonal, coincidiendo con el planteamiento de autores como Edlund (1967), Mackamul (1969), Schafer, (1983), Molina (2008) o Sierra (2008), entre otros, que han propuesto distintos métodos de Lenguaje Musical desde una perspectiva armónica. Trabajar desde la armonía nos lleva a plantear la enseñanza desde las funciones armónicas y no desde las escalas, ni desde el intervalo o el acorde fuera del contexto melódico-armónico. Se debe buscar ese tópico interiorizado ya por el alumno para poder reconocerlo y ponerle nombre, pero este reconocimiento no debe buscarse sólo en los ejercicios que realice dentro del ámbito escolar, sino más bien en la música que escucha fuera de dicho contexto, que es la que sin duda mayor peso ha ejercido en la adquisición de sus conocimientos previos y en el proceso de aculturación defendido por López de Arenosa (2004). Para llevar a cabo estas ideas es necesario hablar al alumnado sobre la tonalidad, los acordes y las funciones armónicas antes de lo que habitualmente se suele hacer, basándolos en la escucha, la interpretación y la creación, que son los tres ejes que articulan las propuestas defendidas en esta investigación (Malagarriga, Gómez y Viladot, 2013; Pérez, 2011).

El concepto de tonalidad se utiliza principalmente para designar un sistema jerárquico de relaciones entre las alturas, formando melodías y armonías que están guiadas por el elemento más importante, central y estable, llamado tónica. Al respecto, Ponsatí (2011) señala que:

Melodía y armonía no están en absoluto aisladas ni desconectadas, sino que, entre ellas, se establece una interacción constante, hasta el extremo de afirmar que la una puede considerarse una extensión de la otra. (p. 52)

Dowling (1994) destaca que "las notas de una melodía se entienden como un tejido de relaciones contextuales relacionadas con otras notas de la melodía en el marco de una tonalidad y no como estímulos individuales aislados" (p. 149). Por ello, las melodías son más fácilmente reconocidas y retenidas si se presentan en un contexto tonal asequible. Dicha idea es reforzada por Hargreaves (2002) al considerar que los oyentes, mientras escuchan música, van generando expectativas de lo que pasará en la melodía, el ritmo, el timbre, la armonía y, en definitiva, en todos los aspectos formales de la música. Loui y Wessel (2007) se refieren concretamente al significado de la expresión expectativas armónicas como la "anticipación de los efectos psicológicos de los modelos de progresiones de acordes" (p. 1.084). Estas expectativas 
armónicas, tal y como demuestran Bigand, McAdams y Forêt (2000), se presentan tanto en los individuos con formación musical como en aquellos que no han recibido ninguna instrucción. No obstante, Meyer (2005) destaca que la experiencia musical está directamente relacionada con la resolución de estas expectativas.

Desde una perspectiva cognitiva, Despins (2013) considera que la música es el medio más eficaz para desarrollar e incrementar el intercambio de información entre los dos hemisferios cerebrales porque, mientras hay actividades que requieren la acción del hemisferio izquierdo para regular la secuencia temporal y serial, simultáneamente se produce otra actividad relacionada con la expresión emocional que viene regulada por el hemisferio derecho. En este sentido, Arias (2007) afirma que el aspecto melódico pertenece al hemisferio derecho, mientras que el izquierdo tiene un componente más analítico, lo expresó de esta manera: "El hemisferio derecho es el especializado en el aspecto más holístico de la música y el izquierdo en el analítico" (p. 32). Otros autores como Altenmüller, Gruhn, Parntz y Liebert (2000) también han observado que durante la ejecución musical se activan diferentes áreas del córtex cerebral al mismo tiempo.

En el reconocimiento de las cualidades de los sonidos musicales, Levitin (2006) apunta la intervención de dos procesos cognitivos:

- Mecanismos sensoriales que gobiernan cómo se traduce la energía física del sonido en el ambiente a los impulsos eléctricos utilizados por el cerebro (proceso "bottom-up").

- Mecanismos perceptuales cognitivos que gobiernan cómo identificamos e interpretamos los sonidos (proceso "top-down”).

Ambos mecanismos sensoriales y perceptuales se informan el uno al otro de forma continua (Loebach, Conway y Pisoni, 2009). Por otra parte, diversos estudios consideran que la comprensión musical se fundamenta en dos grandes universos: la configuración métrica y la configuración tonal. Malbrán (2007) lo argumenta diciendo:

Los investigadores en cognición musical consideran las jerarquías métrica y tonal como los grandes organizadores de la comprensión musical. Adscribir la configuración métrica y tonal a un sistema internamente consistente es una herramienta fundamental para analizar auditivamente la música tonal. (p. 7)

En relación con la estabilidad de algunas notas, acordes o regiones tonales, la psicología de la música ha validado experimentalmente diversas hipótesis propuestas por la teoría musical (Bharucha, 1984; Krumhansl y Kessler, 1982). Los resultados indican que, en un contexto tonal de referencia, determinados elementos se perciben más compatibles o estables que otros (Schönberg, 1969). Los elementos menos compatibles o inestables tienen la necesidad de resolver hacia un elemento de mayor estabilidad: el centro tonal. Por tanto, la percepción y elaboración de las relaciones tonales compromete al auditor en una serie de procesos mentales por medio de los cuales las relaciones entre los sonidos de la obra musical -desde una perspectiva psicológica- se representan en la mente de un modo jerárquico. Dicha jerarquía puede explicarse en términos de que la mente atribuye a los sonidos una importancia y una posición relativa dentro del sistema tonal, a partir de la adjudicación de una tónica (Krumhansl, 2004). 
Acerca de la melodía, las investigaciones indican que el oyente supone ciertas estructuras armónicas a partir del material melódico del que dispone (Platt y Racine, 1994). En cuanto a la armonía, Butler (1989) opina que afecta directamente a la memorización de melodías y, también, a los criterios sobre la tonalidad.

Patel (2012) muestra que hay una integración estructural entre la música y el lenguaje, de forma que ambos comparten los recursos neuronales que posibilitan la activación de las redes donde reside la representación de estos campos específicos.

Finalmente, tal y como señala Lasuén (2014), cabe destacar que en los últimos años han aparecido distintos recursos didácticos que utilizan ejemplos de muy diversa procedencia -músicas populares, urbanas, folclore, etc.- para explicar conceptos de armonía tonal. Uno de los más consolidados es el libro The Musician's Guide to Theory and Analysis (Clendinning y Marvin, 2011), pero también destacan los propuestos por Roig-Francolí (2011) o Laitz (2012). Pensando en las aplicaciones de los conceptos armónicos para la enseñanza-aprendizaje, Costa-Giomi (2001) concluye que su adquisición no parece ser efectiva antes de los 9 años de edad. A pesar de todo, la autora sugiere que, mucho antes, los niños pueden emplear estos elementos en actividades imitativas y experimentales, los cuales, alcanzada la edad adecuada, podrán ser utilizados para reforzar los conceptos armónicos.

\section{Metodología de la investigación}

\subsection{Método de investigación utilizado}

Se trata de un proyecto de investigación realizado por una profesora en su aula en colaboración con otros dos expertos en Pedagogía, que se centra en el estudio de la intervención sobre determinados grupos de alumnos, utilizando básicamente métodos cualitativos.

Taylor y Bogdan (1986) definen la investigación cualitativa como "aquella investigación que produce datos descriptivos por medio de las palabras y la conducta observable" (p. 20). En la misma línea, Barba, González-Calvo y Barba-Martín (2014) afirman que "se trata de una propuesta para comprender o transformar los acontecimientos, las normas y los contextos desde la propia perspectiva de los participantes" (p. 3).

En el presente trabajo se ha optado por realizar una investigación cualitativa porque lo que se pretende es comprender a las personas dentro de su marco de referencia (Taylor y Bodgan, 1986); el investigador se convierte en el principal instrumento de recogida de datos (Anguera et al., 1998); la investigación se orienta a comprender e interpretar lo singular de los fenómenos sociales, dejando las explicaciones de las leyes generales a las ciencias nomotéticas (Albert Gómez, 2006); hay una preocupación directa por la experiencia tal y como es vivida, sentida o experimentada (Sherman y Webb, 1988); el foco de la investigación tiene carácter exploratorio y descriptivo (Maykut y Morehouse, 1994); se prefiere la teoría nacida de los datos sobre sí mismos (Gimeno y Pérez, 1985); se utiliza un lenguaje expresivo que recoja los matices específicos de cada fenómeno analizado (Eisner, 1991); y se recurre a distintas técnicas de recogida de datos para aumentar la validez de las conclusiones (Goetzy LeCompte, 1988). 
El método de investigación utilizado ha sido la investigación-acción, pero entendida como investigación en el aula, ya que no ha habido varios ciclos de intervención (Barba, González-Calvo y Barba-Martín, 2014). Se trata de un estudio participativo en el que se transforma la realidad mientras se investiga, de tal manera que las características principales de la investigación en el aula (Elliot, 1990; McKernan, 1999) en el trabajo realizado se concretan en tres: a) el docente se convierte en el investigador de su propia práctica; b) el estudio de campo mantiene una perspectiva práctica, ya que la acción ha sido guiada por la recogida de datos; y c) la unidad entre la práctica y el proceso de investigación se ha mantenido constante. Asimismo, entre las características que compartimos con Cain (2013) destacamos que es un tipo de investigación en la que tanto los estudiantes como los profesores trabajan juntos para mejorar el aprendizaje y que no se trabaja con muestras representativas, sino que los profesores investigadores trabajan con sus propios estudiantes. La investigación de los propios docentes sobre sus prácticas educativas siguiendo estos mismos planteamientos es cada vez más frecuente, destacando trabajos como los de Bisquerra (2004) y Kincheloe (2012).

En el contexto de la educación musical, Jimeno (2000) apunta que los resultados obtenidos a través de la investigación-acción se generalizan con prudencia porque los datos tomados son parciales, de naturaleza e interpretación cualitativa, y los resultados están ligados a la situación contextual. No obstante, estos riesgos son, al mismo tiempo, virtudes, ya que ayudan a descubrir un alumno/a real, intentando crear situaciones cada vez más ricas y complejas.

Por otra parte, cabe señalar que, a pesar de que se ha realizado una investigación cualitativa, algunos de los datos recogidos han requerido cuantificaciones precisas (calificaciones de los alumnos y porcentajes en las respuestas ofrecidas en los cuestionarios cerrados), pero la interpretación posterior que se ha hecho de los mismos ha sido cualitativa. Esta decisión se debe a que el interés de la investigación radicaba en realizar un análisis interpretativo que permitiera llegar a conclusiones sobre la utilidad de las propuestas didácticas presentadas, no en realizar una comparación minuciosa con una situación de aprendizaje anterior.

Por lo tanto, se ha elaborado un proceso metodológico peculiar, adaptado a los objetivos y a la estructura de la investigación. Todo ello sugiere el carácter versátil, flexible, creativo y adaptable del método de investigación al objeto de estudio.

\subsection{Selección de los participantes}

El estudio se ha llevado a cabo con 79 alumnos del Conservatorio Profesional de Música de Segovia, pertenecientes a siete grupos de Lenguaje Musical de los dos primeros cursos de las enseñanzas elementales. Resulta conveniente aclarar que se buscaba comprender en profundidad todo lo que ocurría en las aulas concretas en las que se estaba desarrollando la investigación.

Además, para la obtención de los distintos datos necesarios para la investigación, los cuales se describen más adelante, se ha contado con la colaboración de:

- Padres y madres (en adelante se utilizará la palabra genérica "padres" para hacer referencia a ambos) de los alumnos de la profesora-investigadora. Todos han participado en la investigación aportando datos a través de las tutorías y 
los correos electrónicos que dirigían a la profesora durante el curso académico. No obstante, algunos han participado también de forma más directa a través de entrevistas individuales (cuatro familiares de primero y tres de segundo) y grupales (seis familiares de primero y siete de segundo).

- Cinco profesores especialistas de instrumento que eran tutores de los alumnos que asistían a clase de Lenguaje Musical con la profesora-investigadora, entre los cuales se encontraban dos miembros del equipo directivo del conservatorio.

\subsection{Técnicas e instrumentos para la recogida de datos}

Para la obtención de los datos de la investigación, se han combinado distintas técnicas e instrumentos, lo cual ha permitido realizar su triangulación y aportar mayor validez y credibilidad a los resultados (Barba, 2013). Dichas técnicas e instrumentos han sido los siguientes:

- Diario de la profesora.

- Entrevistas semiestructuradas en las que la profesora-investigadora planteó una serie de cuestiones pero que estaban abiertas a otras aportaciones no determinadas previamente, ya que las respuestas dadas por las personas entrevistadas provocaban nuevas preguntas para clarificar los temas tratados. Se realizaron dos tipos de entrevistas:

- Entrevistas individuales a padres y profesores de instrumento (tutores) de los alumnos a mitad de curso, en el mes de febrero (modelos en anexo I).

- Entrevistas grupales a padres y a los distintos grupos de alumnos a final de curso, en el mes de junio (modelo en anexo II).

- Cuestionarios abiertos y cerrados cumplimentados por los alumnos a mitad y a final de curso, respectivamente (modelos en anexos III y IV). Cabe señalar que, a pesar de que la utilización de cuestionarios es más característica del enfoque cuantitativo, en esta investigación se le ha dado un enfoque cualitativo.

- Análisis de documentos:

- Correos electrónicos enviados por los alumnos o sus padres.

- Trabajos y exámenes de los alumnos.

- Videos y fotografías con las actividades de los alumnos.

\section{Descripción de la intervención educativa para trabajar el sistema tonal- armónico}

En el presente trabajo se han utilizado distintas estrategias didácticas que se describen a continuación. Todas ellas se han desarrollado en el aula de forma conjunta a lo largo de un curso académico, desde septiembre hasta junio.

\subsection{Identificación auditiva de los acordes tonales}

Los alumnos debían identificar auditivamente series de acordes que iba tocando la profesora en el piano, representándolos corporalmente de esta manera: levantando 
los brazos cuando lo que escuchaban les producía sensación de tensión (dominante), bajándolos cuando sentían reposo (tónica) o dejándolos en cruz delante del pecho cuando la sensación percibida se encontraba a medio camino entre ambos (subdominante). En un primer momento, los acordes se interpretaban en una única posición, pero progresivamente se fue aumentando la dificultad cambiándolos tanto de posición como de octava (video 1).

\subsection{Reconocimiento auditivo de intervalos}

Se trataba de identificar auditivamente distintos intervalos, utilizando dos tipos de estrategias:

- Escalas y arpegios: Si las dos notas sonaban como el principio de una escala se trataba de un intervalo de segunda y, si sonaban como un arpegio, podían ser terceras (si eran las dos primeras notas) o quintas (si eran la primera nota y la tercera).

- Comienzo de distintas canciones: En el segundo curso, se utilizaron también canciones que comenzaban con cada uno de los intervalos. Por ejemplo, se asociaba el intervalo de cuarta justa con el comienzo de la canción Pinocho fue a pescar.

\subsection{Entonación memorística de escalas y arpegios}

Se diseñaron ejercicios específicos para el calentamiento de la voz y la preparación del oído, los cuales debían cantar los alumnos de memoria antes de la interpretación de canciones o lecciones de entonación y de la escritura de canciones o dictados musicales. Dichos ejercicios estaban dirigidos únicamente a los alumnos de segundo (con los de primero se utilizaba sólo la fononimia de Kodály) y se basaban en las escalas y arpegios de las tonalidades trabajadas, es decir, do mayor, la menor y sol mayor, incluyendo notas de adorno en los dos últimos. Para poder trabajarlos en casa hasta conseguir memorizarlos, los alumnos disponían de una plantilla con los ejercicios y unos audios con su interpretación (anexo $\mathrm{V}$ y audio 1 ).

\subsection{Invención y adaptación de motivos rítmico-melódicos}

Un alumno proponía un motivo eligiendo las notas reales que quisiera del acorde de tónica y cantándolas con un ritmo también escogido por él. Después, el resto de la clase debía repetirlo y adaptarlo de memoria a las notas de los demás acordes que se estuvieran utilizando. Se repetía dos veces el motivo propuesto en cada acorde para facilitar el control y la asimilación de la actividad y siempre se terminaba con el acorde de tónica, haciendo una nota larga en la segunda repetición para que, al frenar el ritmo, sonara más conclusivo. La profesora acompañaba las interpretaciones de los alumnos tocando los acordes en el piano, pero no la melodía. Al principio, se empleaban únicamente notas reales presentes en los acordes (video 2), pero, progresivamente, se fueron añadiendo también notas de adorno: notas de paso, floreos y apoyaturas (video 3). En el anexo VI se muestra cómo se adaptarían varios motivos 
con notas reales o de adorno a distintos acordes, para formar frases musicales de 8 compases.

\subsection{Aprendizaje, análisis y transcripción de canciones}

Una vez al mes, se trabajaba en clase con canciones que los alumnos debían transcribir al dictado, al mismo tiempo que se realizaba un análisis de las mismas (ritmo, notas, giros melódicos, estructura formal, etc.). Al realizar esta actividad, se insistía a los alumnos en que debían atribuir un sentido a todo lo que escribieran, lo cual significa que todas las notas que pusieran tenían que encontrarse en el acorde de debajo y, si alguna no lo estaba, debían poder explicarla como floreo, nota de paso o apoyatura porque, de no ser así, se trataba de un error. A modo de ejemplo, en el anexo VII se expone la canción popular "Debajo un botón" escrita por un alumno de segundo.

\subsection{La principal aportación: la creación de canciones}

A partir del trabajo que se acaba de describir en el apartado anterior, los alumnos debían componer sus propias canciones en casa, siguiendo las mismas pautas y utilizando el programa informático de edición de partituras MuseScore, como puede observarse en el anexo VIII. Una vez realizadas, se las enviaban a la profesora por correo electrónico para su corrección. Los errores cometidos se indicaban de la siguiente manera:

- Las notas que no se encontraban en el acorde y no se podían explicar como notas de adorno se marcaban en rojo.

- Las palabras que sonaban mal porque los acentos lingüísticos no coincidían con los musicales se marcaban en negrita.

- Si no se había respetado alguna pauta (partes, compases con la misma línea melódica, notas a utilizar, etc.) se indicaba debajo en forma de observaciones.

Todo ello puede observarse en el ejemplo recogido en el anexo IX. En estos casos, debían corregirla y enviar una nueva versión, como muestra el anexo X.

Cuando la canción era correcta, debían aprendérsela de memoria para cantarla en clase, tanto con notas, lo cual ayudaba a que fijaran los sonidos en su mente, como con letra. Asimismo, podían acompañar su actuación con instrumentos, dibujos, disfraces o todo aquello que consideraran conveniente para enriquecer la puesta en escena (video 4). Durante la interpretación, las canciones se proyectaban en la pared para poder comentarlas posteriormente (fotografías 1 y 2).

\section{Análisis de los datos obtenidos}

Para el análisis de los datos y la exposición de los resultados obtenidos, se han establecido tres categorías de análisis: motivación hacia las actividades armónicas, utilidad del conocimiento armónico y dificultades encontradas.

Antes de abordar dichas categorías, se considera oportuno apuntar que en el posterior análisis se han tenido en cuenta los procesos cognitivos de mejora del apren- 
dizaje (Rivas, 2008), especialmente los procesos que posibilitan la concentración en las distintas actividades musicales, la imitación de prácticas observadas y el desarrollo de capacidades de percepción y memoria auditiva.

Para identificar los instrumentos/técnicas y las personas de las que se ha extraído la información se han utilizado los códigos recogidos en la tabla 2, garantizando el anonimato de los participantes. La codificación se ha realizado con el programa informático Atlas-ti.

Tabla 1. Codificación de los documentos y de las personas participantes

\begin{tabular}{|l|l|l|l|l|l|l|l|}
\hline $\begin{array}{l}\text { Instrumentos } \\
\text { técnicas }\end{array}$ & Código & Personas & Código & $\begin{array}{l}\text { Curso/ } \\
\text { Grupo }\end{array}$ & Mes* & $\begin{array}{l}\text { Ejemplo } \\
\text { de código }\end{array}$ & $\begin{array}{l}\text { Significado } \\
\text { del código }\end{array}$ \\
\hline Diario & D & $\begin{array}{l}\text { Profesora } \\
\text { investiga- } \\
\text { dora }\end{array}$ & P & & Enero & DPE & $\begin{array}{l}\text { Diario de la profe- } \\
\text { sora investigadora, } \\
\text { de enero }\end{array}$ \\
\hline $\begin{array}{l}\text { Correo electró- } \\
\text { nico }\end{array}$ & CE & Familiar & F & $1 \mathrm{C}$ & Mayo & CE1CF4Y & $\begin{array}{l}\text { Correo electrónico } \\
\text { del primer curso, } \\
\text { de un familiar del } \\
\text { alumno número 4, } \\
\text { de mayo }\end{array}$ \\
\hline $\begin{array}{l}\text { Cuestionario } \\
\text { abierto }\end{array}$ & C & Alumno & A & $2 \mathrm{C}$ & & CA2C & $\begin{array}{l}\text { Cuestionario abier- } \\
\text { to de un alumno del } \\
\text { segundo curso** }\end{array}$ \\
\hline $\begin{array}{l}\text { Entrevista } \\
\text { individual }\end{array}$ & E & $\begin{array}{l}\text { Profesor- } \\
\text { Tutor }\end{array}$ & T & & & ET6 & $\begin{array}{l}\text { Entrevista al profe- } \\
\text { sor-tutor 3 }\end{array}$ \\
\hline $\begin{array}{l}\text { Entrevista gru- } \\
\text { pal }\end{array}$ & EG & Alumnos & As & $2 \mathrm{CB}$ & & EG2CAsB & $\begin{array}{l}\text { Entrevista grupal a } \\
\text { alumnos de segun- } \\
\text { do curso, del grupo } \\
\text { B }\end{array}$ \\
\hline
\end{tabular}

${ }^{*}$ Se utiliza la inicial del mes, excepto en mayo (Y) para diferenciarlo de marzo (M).

${ }^{* *}$ Los cuestionarios los cumplimentaron de forma anónima, por lo que sólo se puede indicar el curso del alumno.

\subsection{Motivación hacia las actividades armónicas}

Muñoz y Bonastre (2013) apuntan que la expresividad debe mejorarse en los procesos de enseñanza-aprendizaje de la música y que la inclusión de elementos explícitos de entrenamiento de la expresividad emocional en el currículo de los conservatorios resulta positivo para completar la formación de los alumnos.

En este sentido, se ha observado que el trabajo creativo de invención y adaptación de motivos a partir de distintas secuencias armónicas ha sido muy gratificante para los alumnos, tal como se constata en los siguientes fragmentos de entrevistas:

A34- A mí los motivos me han parecido divertidos, porque todos teníamos la oportunidad de inventarnos nuestra propia canción, solo que más pequeño. (EG1CAsC, p. 16) 
El hecho de inventar después letras diferentes para dichos motivos, haciendo coincidir los acentos lingüísticos con los musicales, también les ha resultado interesante.

En varios grupos me han pedido que lea en voz alta las letras que se han inventado, porque la parte creativa es lo que más les gusta. (DPF, p. 2)

Estos resultados coinciden con las respuestas ofrecidas por 1.635 alumnos de distintos conservatorios españoles a los cuestionarios utilizados en el estudio de Berrón (2015), las cuales muestran que los estudiantes no solo disfrutan interpretando música escrita por compositores de diferentes épocas y estilos artísticos, sino que también existe en ellos un gran deseo por expresarse a través de su propia música. Por ello, se considera inadecuado que, en general, se tenga poco presente en las aulas el deseo expresivo y creativo de los alumnos y que en pocas ocasiones se les dé la oportunidad de iniciarse en la composición desde el principio, posponiéndolo hasta los estudios de armonía de las enseñanzas profesionales.

La Ley Orgánica 8/2013, de 9 de diciembre, para la Mejora de la Calidad Educativa (LOMCE) apunta la necesidad de generar curiosidad en el alumnado a través de propuestas creativas que faciliten el desarrollo de su metacognición. Al respecto, se ha comprobado que la creación de sus propias canciones era una actividad motivadora para el $82 \%$ de alumnos (ver gráfica 1 con los resultados de los cuestionarios en el anexo XI). Convertirse en compositores les hacía "sentirse importantes", dueños de su propio aprendizaje autónomo, lo cual resultaba muy beneficioso para su implicación en el proceso educativo. Así lo reflejaban las palabras de una madre y un alumno:

F37- El tema de la composición es lo que a ella le ha enganchado porque, desde muy pequeña, se siente muy importante diciendo: “¡Esto lo he hecho yo y encima sé cómo!” (EG1CFs, p. 11)

Me gusta mucho hacer composiciones y luego cantarlas en clase. (CA2C, pre. 4)

Se sienten orgullosos del trabajo realizado por ellos mismos y disfrutan compartiéndolo con los demás, ya sean sus familiares, compañeros, amigos o profesores. El amor propio y el espíritu de sacrificio y esfuerzo están unidos en pro de una mejora de su propio desarrollo creativo.

T- Hay algunas veces que después de tu clase vienen a la mía y vienen como ilusionados: quieren cantarme su canción, enseñarme su canción que han compuesto. (ET2, p. 5)

Me ha gustado mucho componerla y estoy deseando que la veas. (CE1CA4E, p. 2)

La puesta en escena para presentar su trabajo era fundamental para ellos, por lo que había que prepararla bien con todo aquello que cada cual estimara conveniente, permitiéndoles hacer uso nuevamente de su creatividad. Otro ejemplo claro se encuentra en los comentarios realizados por una alumna y por un padre en los fragmentos de entrevistas que recogemos a continuación: 
A37- Estoy deseosa de cantar la canción, de enseñar a mis compañeros el trabajo y de hacerlo con un acompañamiento y con nuestros trabajos, nuestros dibujos. (EG1CAsF, p. 24)

F- La puesta en escena para ella es vital y dice: “¡Me tienes que preparar algo!”. Tiene mucha emoción por esa representación. El que se reconozca su trabajo es importante. (EF6, p. 9)

Dichos comentarios coinciden con las respuestas ofrecidas por los alumnos en los cuestionarios cumplimentados a final de curso, ya que el 79\% afirmó que era bueno presentar las canciones en clase para que las conocieran sus compañeros (gráfica 9 del anexo XI) y el 76\% manifestó que se divertía interpretando las canciones creadas (gráfica 2 del anexo XI), sirviendo de estímulo para el aprendizaje. Incluso, el 58\% indicó que consideraba interesante presentar las canciones en una audición abierta al público (gráfica 10 del anexo XI).

El hecho de utilizar un programa informático para realizar sus canciones constituyó un acercamiento con su entorno más cercano en relación a las TIC, un aliciente, ya que les permitía escuchar lo que iban escribiendo.

A24- A mí me gusta más hacerlas por el ordenador y luego las saco, porque, ya de paso, va sonando mientras escribo. (EG1CAsC, p. 14)

F- Le gusta ponerse en el programa e ir viendo cómo suena: le ayuda mucho. (E1CF2, p. 6)

Las actividades compositivas han resultado tan motivadoras que, incluso, algunos alumnos dedicaron parte de su tiempo libre a componer por iniciativa propia, tal y como expresaron un padre y una alumna:

F70- Lo del ordenador para mí es un acierto, porque hay veces que ella, incluso, se pone a componer sin que se lo pidas como tarea. (EG2CFs, pp. 17-18)

Me he inventado una canción con el MuseScore para hacerla con viola y cantando, se la enviaré también a mi profesor de instrumento. Espero que te guste. (CE2CA56M, p. 2)

Se considera fundamental cuidar la motivación para favorecer el aprendizaje, ya que, aunque la música tiene un elevado componente lúdico y se asocia al entretenimiento y a la diversión, su aprendizaje es largo y costoso, demandando gran constancia y esfuerzo. Por ello, si en los estudios musicales de los conservatorios no se cuida el fomento de la motivación interna de los alumnos, muchos pueden terminar abandonándolos. En todo momento, se ha perseguido generar en los alumnos expectativas positivas de eficacia (evaluación sobre las propias capacidades para llevar a cabo la tarea) y de resultados (evaluación sobre las posibilidades de tener éxito). 


\subsection{Utilidad del conocimiento armónico}

La utilidad de las propuestas aplicadas se evidencia, en parte, al analizar las calificaciones obtenidas por los alumnos a final de curso, derivadas del compendio de herramientas de evaluación utilizadas dentro del proceso de evaluación formativa desarrollado a lo largo del mismo, en el que participaron tanto la profesora investigadora como los demás miembros del departamento de Lenguaje Musical. Tal y como muestran las gráficas del anexo XII, en el primer curso de las enseñanzas elementales no ha habido ningún suspenso, el $29 \%$ ha obtenido aprobado-bien, el $32 \%$ notable y el 39\% sobresaliente. Por su parte, en el segundo curso ha suspendido el $8 \%$ de los alumnos, el $21 \%$ ha conseguido aprobado-bien, el $50 \%$ notable y el $21 \%$ sobresaliente. Estos datos han sido obtenidos a través de las pruebas prácticas y escritas realizadas en los distintos apartados en los que se estructura el Lenguaje Musical: ritmo, entonación, teoría y dictado, a partir de las cuales se obtiene la nota final de la asignatura. Para una comprensión más amplia de estos datos pueden consultarse las gráficas del anexo XIII, que muestran de forma más específica las calificaciones en cada uno de los cuatro apartados tradicionales de la asignatura de Lenguaje Musical.

Dichos resultados apoyan el convencimiento de que todo lo que los alumnos crean y manipulan por sí mismos lo aprenden y memorizan mejor, en lo cual coinciden reconocidos autores (Bautista, Pérez-Echeverría y Pozo, 2011; López-Íñiguez, Pozo y De Dios, 2014; Torrado y Pozo, 2006). En este sentido, el aprendizaje significativo parte de generar entre el alumnado la curiosidad por aprender y por desarrollar su creatividad dentro de la clase de música.

A61- Lo que te inventas tú, como que se te queda mejor en la cabeza. (EG2CAsB, p. 23)

De hecho, el 97\% de los alumnos también reconoció que la creación de canciones era interesante para su formación musical (gráfica 3 del anexo XI) y el 94\% afirmó que todos los músicos deberían saber hacerlas (gráfica 4 del anexo XI).

Pero, para que toda improvisación o creación musical tenga coherencia, se defiende partir siempre de un análisis previo (en nuestro caso, de canciones breves), lo cual ha permitido que los alumnos pudieran conocer los elementos constituyentes de la música que luego iban a manipular. Los profesores de instrumento de los alumnos aprobaban esta forma de proceder.

T- Me parece muy adecuada la forma de: "Bueno, este es el sistema de existir de la música, ahora lo vas a hacer tú”. (ET4, p. 3)

T- La creatividad implica, primero, tener un conocimiento amplio del campo, o sea, tú no puedes innovar en algo si no conoces el lenguaje. (ET1, p. 13)

Al respecto, resulta destacable el hecho de que, al transcribir de oído o componer canciones, los alumnos debían justificar cada una de las notas que escribían, bien como notas reales del acorde o como alguna de las notas de adorno estudiadas. Con esto se ha conseguido aumentar su concentración y su capacidad reflexiva, al tiempo que interiorizaban las reglas de la armonía tonal. 
F- Lo tiene super claro: “¡No, no!, es que aquí esta nota no puede ir, mamá, porque fíjate aquí abajo lo que tienes puesto, y aquí no puede ir esta otra nota, aunque tú la quieras meter. (E1CF2, p. 6)

Otro aspecto fundamental es que el alumnado ha manifestado sentirse más cómodo transcribiendo de oído canciones una vez que habían identificado su estructura armónica, la cual les servía de guía, que realizando dictados tradicionales en los que no había ninguna referencia de acordes.

A28- En las canciones reconocía mejor los sonidos ayudándome de los acordes que tocabas con el piano. (EG1CAsC, p. 21)

A36- Para mí son más difíciles los dictados, porque no tienes la guía de los acordes. Tienes que identificar las notas sin tener ninguna guía que te diga si es una u otra. (EG1CAsF, p. 19)

Además, era interesante comprobar cómo lo que aprendían en las canciones lo aplicaban también de forma espontánea al resolver dictados tradicionales, ya que tenían el impulso de buscar la armonía subyacente a la melodía, identificando notas reales, de paso, floreos y apoyaturas, lo cual indica que iban analizando internamente los acordes presentes en cada compás. Ello demuestra que, cuando se comprenden los fundamentos que justifican la construcción de melodías dentro de nuestro contexto tonal-armónico, se reconocen los elementos constituyentes aunque no estén los acordes escritos debajo.

A69- En los dictados, si te resulta más fácil, puedes averiguar un poco el acorde y saber si la nota te entra en el compás o qué nota te puede entrar .(EG2CAsD, p. 21)

Yo les insistía en lo gratificante que había sido para mí realizar un dictado tradicional con ellos y preguntarles: “¿Os ha salido algún floreo o nota de paso?”, y que dijeran rápidamente: "Sí", lo cual manifiesta que, incluso sin tener los acordes dibujados debajo, empiezan a reconocer la armonía presente en cada compás y los elementos que configuran la melodía, dotándoles de significado, lo cual supone un nivel de comprensión de la música mucho mayor que la simple lectura de notas y ritmos. (DPD, p. 11)

El hecho de interpretar, posteriormente, las canciones que habían creado ante sus compañeros, les ha ayudado a mejorar su autoestima, es decir, a ganar confianza y seguridad en sí mismos.

A41- Yo me siento bien. No tengo nada de vergüenza, porque estoy acostumbrado a que mis compañeros me oigan. (EG1CAsF, p. 24)

A7- Me gusta venir a cantarlas porque sé que luego mis compañeros me van a aplaudir y eso me encanta. (EG1CAsA, p. 8)

Y, sobre todo, a comprender mejor la música y a fomentar su creatividad, como muestran los fragmentos de entrevistas que cierran este epígrafe: 
T- Es genial, porque luego llegan a una obra y pueden ver las mismas cosas que en las que ellos componen. [...] Creo que es estupendo que compongan y que sean creativos. (ET5, p. 3)

A75- A mí me resulta más fácil con acordes porque con acordes, si tienes notas reales, pues, si sabes los acordes que son, tienes que poner las notas de esos acordes, no te las puedes inventar.

$\mathrm{P}-i \mathrm{Y}$ si no son notas reales?

A75- Pues tiene que ser un floreo, una nota de paso o alguna de esas y entonces tienes que fijarte bien en los acordes porque si tú pones otra nota que no es ni floreo, ni nota de paso, ni apoyatura, pues, como dice A71, si le das al play suena mal y, entonces, lo tienes que cambiar, porque suena mal. (EG2CAsD, p. 13)

\subsection{Dificultades encontradas}

En general, las actividades armónicas que se han desarrollado en el aula han sido bien entendidas y realizadas por los alumnos; prueba de ello es la siguiente transcripción:

A48- Reconocer los acordes de tónica, subdominante y dominante es sencillo. (EG2CAsA, p. 4)

P- Cuando os habéis inventado algún motivo y, después, teníais que irlo pasando de memoria a los distintos acordes, ¿de eso qué me decís?

A21- Eso es fácil.

A18- Sí, es fácil. (EG1CAsB, p. 21)

A75- Una vez que te has escuchado las canciones, ya las tienes en la memoria y las puedes escribir fácilmente, porque los acordes te ayudan. (EG2CAsD, p. 4)

La creación de canciones fue la única actividad que, al principio, les resultó más complicada, porque les surgían muchas dudas acerca de las pautas a seguir y de cómo utilizar el programa informático MuseScore, olvidando la musicalidad. En el diario de la profesora e investigadora, se encuentran las siguientes reflexiones durante el mes de octubre:

Para ser la primera canción que se inventan, no les ha salido muy mal, pero han cometido bastantes errores por despistes: utilizaban notas o figuras que no aparecían en las pautas, olvidaban poner el compositor o las funciones de los acordes, nombraban mal el archivo que me tenían que mandar, utilizaban palabras que no sonaban bien por los acentos... (DPO, p. 8)

Parecía que habían ido rellenando los compases siguiendo las pautas de una forma artificial, sin tener en cuenta si aquello sonaba bien y era fácilmente cantable. Por tanto, para la próxima canción, trabajaremos más en clase los aspectos formales y las estrategias para componer con sencillez y musicalidad. (DPO, p. 10) 
Por ello, se considera que siempre que se demanda a los alumnos realizar algún tipo de actividad creativa es necesario proporcionarles pautas claras y sencillas que les permitan mantener el control sobre lo que están haciendo, especialmente en los primeros cursos (Hernández, Hernández y Milán, 2007). Así lo recogía la profesora en su diario:

Para casa les he mandado inventar su propia canción siguiendo las mismas pautas recogidas en las canciones que habíamos aprendido en clase: notas, figuras, acordes, etc. (DPO, p. 8)

Considero que todos estos errores no son síntoma de que la tarea no se haya entendido o sea complicada para ellos, sino que se deben a una falta de atención o rigurosidad a la hora de seguir las pautas. (DPO, p. 8)

La necesidad de ofrecer unas pautas claras como punto de referencia para la creación musical era reconocida por los distintos miembros de la comunidad educativa.

T- Si les dices: "Crea de la nada", no saben, no tienen referencias. Tener un molde como punto de partida me parece que es muy interesante y práctico. (ET2, p. 10)

F- Tener que componer canciones cortitas, pero con unas pautas determinadas, les encauza mucho el trabajo y les da una sistematicidad en la forma de trabajar. A mí me parece bien. (E2CF45, p. 6)

Además, cuando se plantean actividades entretenidas y atrayentes para los alumnos, la sensación de dificultad desaparece, a pesar de que exijan un elevado nivel de concentración y sistematicidad (Cabrera, 2010; Puente-Ferreras, 2003).

T- No lo veo complejo. Veo que ellos entienden lo que tienen que hacer y que les divierte. La verdad es que me parece muy atractivo para los chicos de esta edad y, desde fuera, se ve un trabajo con un fundamento y bien hecho. (ET3, p. 7)

De esta forma, pronto se familiarizaron con la actividad y disminuyeron las dificultades al realizar sus composiciones.

Esta vez han seguido mejor las pautas y la verdad es que, en general, estaban bastante bien, ihe notado mucha mejoría y me he alegrado muchísimo! Se aprecia más musicalidad. (DPN, p. 3)

A2- Antes me liaba un poco, porque no sabía ni los nombres ni cómo sonaban, pero ahora ya lo llevo muy bien y ya sé diferenciarlos y lo que son. (EG1CAsA, p. 11)

En los cuestionarios respondidos por los alumnos a final de curso, el 94\% indicaron que utilizar un programa informático les había facilitado realizar las canciones (gráfica 6 del anexo XI), por lo que se considera que fue un acierto incluir las nuevas tecnologías.

F- Ahora le veo suelto. El programa lo maneja muy bien. (E2CF45, p. 5) 
A6- Al principio, las canciones del ordenador me parecían un poco difíciles, pero luego ya cogí cómo se hacían y ahora me gusta mucho crear canciones, porque me divierto. (EG1CAsA, p. 8)

Por todo lo expuesto, a pesar de las dificultades iniciales y de que el $44 \%$ de los alumnos consideró que realizar composiciones implicaba cierta complejidad (gráfica 5 del anexo XI), el 82\% respondió que les gustaría seguir realizando esta actividad (gráfica 8 del anexo XI) e, incluso, el 41\% afirmaron que les gustaría componer canciones con más frecuencia (gráfica 7 del anexo XI).

Estos datos permiten afirmar que la percepción de dificultad en las actividades propuestas no tiene por qué interferir en la motivación del alumnado para desear realizarlas, coincidiendo con los resultados obtenidos en estudios previos como los de Kenny (2008), Miguel-Tobal (1990) y Virues (2005). El despertar y mantener viva la motivación dentro del aula ha facilitado una mayor interacción con el alumnado y ha contribuido a que su implicación haya sido alta en todas las actividades propuestas.

\section{Conclusiones}

Mediante las nuevas propuestas didácticas desarrolladas en la asignatura de Lenguaje Musical se buscaba llevar a un plano consciente la base armónica subyacente a cualquier melodía tonal, con el fin de facilitar a los alumnos la comprensión de la lectoescritura musical melódica. A partir de dicho planteamiento, el trabajo de investigación presentado ha tenido como eje vertebrador valorar si el estudio sistemático de la música tonal entre el alumnado de conservatorio es más efectivo y eficaz cuando se trabajan la comprensión, la discriminación y el manejo de los acordes de tónica, subdominante y dominante desde el inicio de sus estudios musicales, utilizando para ello una metodología motivadora y adaptada a sus capacidades y psicología infantil. De forma más específica, en este estudio se planteó determinar la utilidad de una serie de propuestas para mejorar su comprensión musical, así como para aumentar su motivación hacia la asignatura y su creatividad.

Conseguir que los alumnos percibieran el discurso musical en su totalidad y no como la suma de elementos aislados (notas, ritmo, melodía, acompañamiento, etc.) ha sido uno de los aspectos que más se han tenido en cuenta en el presente trabajo. Ello ha exigido presentarles las reglas básicas del sistema tonal-armónico mucho antes de lo que suele hacerse en los actuales planes de estudio, que, en muchas ocasiones, posponen dichos aprendizajes hasta los estudios posteriores de armonía, ya en las enseñanzas profesionales. Dichas reglas armónicas han sido interiorizadas con naturalidad por los alumnos a través de:

- La interpretación y la escucha pautadas, que permiten dirigir la atención hacia los puntos de interés.

- El análisis, que ayuda a comprender cómo se construye la música.

- La creación, que es lo que posibilita realmente la adquisición significativa de todo nuevo lenguaje. 
Estas afirmaciones se han corroborado mediante los datos recogidos a lo largo de toda la investigación, basando el rigor de la misma en la triangulación metodológica de la información obtenida a partir de distintas técnicas e instrumentos.

Con respecto a la interpretación y la escucha, la conclusión que se deriva de la investigación es que tanto la entonación como el dictado musical constituyen procesos mentales complejos que se producen en sentido inverso. La lectura vocal se refiere a la interpretación de los sonidos musicales a partir de los signos escritos en una partitura, mientras que el dictado consiste en transcribir los sonidos escuchados por medio de los signos de escritura correspondientes. En este sentido, es destacable la importancia de cultivar automatismos en la relación sonido-nombre y, para conseguirlos, ha resultado de gran utilidad la entonación memorística de escalas y arpegios, a partir del conocimiento de su relación con los acordes de tónica, subdominante y dominante. Igualmente, cultivar la escucha comprensiva de forma vivencial y lúdica ha permitido a los alumnos interiorizar los distintos grados de tensión o reposo producidos por los acordes tonales, pudiendo identificarlos fácilmente al presentarlos mezclados en secuencias armónicas progresivamente más complejas, tanto en posición fundamental como invertidos y en distintas octavas.

En cuanto al análisis, se ha comprobado la necesidad de dirigir la atención de los alumnos hacia aspectos relevantes del discurso musical que les ayuden a comprender su composición y estructura. Al respecto, se ha observado que el análisis ha proporcionado a los alumnos las herramientas para entender cómo estaba construida la música que escuchaban, cantaban o veían escrita. Asimismo, cabe destacar que conocer las bases del sistema tonal es lo que más les ha ayudado a la comprensión de dicha música, buscando las relaciones existentes entre la melodía y la armonía. Como consecuencia de ello, la investigación determina que el alumnado ha manifestado sentirse más cómodo transcribiendo de oído canciones una vez que habían identificado su estructura armónica, la cual les servía de guía, que realizando dictados tradicionales en los que no había ninguna referencia de acordes.

Por otra parte, la creatividad en la composición musical supone un ejemplo claro de cómo se emplea la capacidad humana para resolver problemas e inventar situaciones variadas y ricas de forma imaginativa, comprendiendo la relación existente entre una melodía y los acordes que la sustentan. El estudio concluye que, a través de la improvisación, la adaptación de pequeños motivos rítmicos o melódicos y la composición de sus propias canciones, los alumnos han desarrollado su capacidad auditiva y su creatividad, al mismo tiempo que interiorizaban reglas básicas que dotaban de significado al discurso musical, desde el punto de vista melódico, armónico y formal. Igualmente, se ha comprobado que los alumnos aplicaban posteriormente dichas reglas de forma espontánea al realizar dictados tradicionales, donde también diferenciaban distintas partes y motivos melódicos y tenían el impulso de buscar la armonía subyacente a la melodía, identificando notas de paso, floreos y apoyaturas, lo cual indica que iban analizando internamente los acordes presentes en cada compás.

Como conclusión final del estudio cabe subrayar que sintetizar es recomponer lo que el análisis ha separado, es decir, integrar todas las conclusiones y análisis parciales en un conjunto coherente que cobra sentido pleno al integrarse como un todo único. En este sentido, la utilidad de las propuestas metodológicas basadas en el sistema tonal-armónico ha quedado demostrada en los testimonios de los alumnos, sus familias y sus profesores, así como en las altas calificaciones obtenidas por los estudiantes en los distintos apartados de la asignatura de Lenguaje Musical, lo cual 
evidencia su óptima formación en un sentido integral. Asimismo, las nuevas propuestas implementadas en el aula han desarrollado en los alumnos otras competencias compositivas y creativas, potenciando su motivación hacia la música, el control sobre las tareas que emprenden y un mayor deseo de superación personal.

\section{Referencias bibliográficas}

Albert Gómez, M. J. (2006). La investigación Educativa. Claves teóricas. Madrid: McGraw-Hill.

Altenmüller, E., Gruhn, W., Parntz, D. y Liebert, G. (2000). The impact of music education on brain networks: Evidence from EEG studies. International Journal of Music Education, 35, 47-53.

Anguera, M. T. et al. (1998). Métodos de investigación en psicología. Madrid: Síntesis.

Arias, M. (2007). Música y neurología. Neurología, 22(1), 39-45.

Berrón, E. (2015). Motivación y dificultades de los alumnos en la asignatura de Lenguaje Musical. En Libro de actas CIMIE15 de AMIE licenciado bajo Creative Commons 4.0 International License. Recuperado de http://amieedu.org/actascimie15/2016/ 06/29/a1/

Berrón, E. (2016). Iniciación a la educación auditiva desde un contexto tonal en la asignatura de Lenguaje Musical. (Tesis doctoral). Valladolid: Universidad de Valladolid. Recuperado de https://uvadoc.uva.es/handle/10324/16793

Barba, J. J. (2013). La investigación cualitativa en educación: en los comienzos del siglo XXI. En A. Maravillas y A. Giráldez (Coords.), Investigación cualitativa en educación musical (pp. 23-38). Madrid: Graó.

Barba, J. J., González-Calvo, G. y Barba-Martín, R. A. (2014). Que la fuerza esté contigo: desvelar el lado oscuro de la investigación en educación. Magis. Revista Internacional de Investigación en Educación, 7(14), 125-140.

Bautista, A., Pérez-Echeverría, M. P. y Pozo, J. I. (2011). Piano teachers' conceptions of assessment. Revista de Educación, 355(1), 443-466.

Bharucha, J. J. (1984). Anchoring effects in music: The resolution of dissonance. Cognitive Psychology, 16, 485-518.

Bigand, E, McAdams, S. y Forêt, S. (2000). Divided attention in music. International Journal of Psychology, 35, 270-278.

Bisquerra, R. (Coord.) (2004). Metodología de la investigación educativa. Madrid: La Muralla.

Butler, D. (1989). Describing the Perception of Tonality in Music: A Critique of the Tonal Hierarchy Theory and a Proposal for a Theory of Intervallic Rivalry. Music Perception, 6(3), 219-242.

Cabrera, M. (2010). El valor de la memoria en la educación y su relación con la inteligencia. Revista Digital Innovación y Experiencias Educativas, 36. Recuperado de http://www. csi-csif.es/andalucia/ modules/mod_ense/revista/pdf/Numero_36/MARIA_CABRERA_1.pdf

Cain, T. (2013). Investigación-acción en educación musical. En M. Díaz y A. Giráldez (Coords), Investigación cualitativa en educación musical (pp. 57-76). Barcelona: Graó.

Clendinning, J. P. y Marvin, E. W. (2011). The Musician's Guide to Theory and Analysis. New York, NY: W. W. Norton.

Costa-Giomi, E. (2001). El desarrollo de la percepción armónica durante la infancia. Cua- 
dernos Interamericanos de Investigación en Educación Musical, 2, 43-56. Recuperado de http://www.journals.unam.mx/ index.php/cem/article/view/7311

Dalcroze, E. J. (1980). Rhythm, music \& education. London: The Dalcroze Society.

DeNora, T. (1986). How is extra-musical meaning possible? Music as a place and space for "work". Sociological Theory, 4(1), 84-94.

Despins, J. P. (2013). La música y el cerebro. Madrid: Gedisa.

Dowling, W. J. (1994). Melodic Contour in Hearing and Remembering Melodies. En R. Aielloy J. A. Sloboda (Eds.), Musical perceptions (pp. 176-190). New York, NY: Oxford University Press.

Edlund, L (1967). Modus Vetus. Sight Singing and Ear-Training in Major/Minor Tonality. Estocolmo: Nordiska Musikförlaget.

Eisner, E. (1991). The Enlightened Eye. Qualitative Inquiry and the Enhancement of Educational Practice. New York, NY: McMillan Publ. Co.

Elliot, J. (1990). La investigación-acción en educación. Madrid: Morata.

Fernández Pérez, M. (1988). La profesionalización del docente. Madrid: Escuela Española.

Furnó, S. (2003). La formación de conceptos musicales. Cuadernos Interamericanos de Investigación en Educación Musical, 6, 81-108. Recuperado de http://revistas.unam.mx/ index.php/cem/article/ view/7335

Gimeno, J. y Pérez, A. (1985). La Enseñanza: Su Teoría y su Práctica. Madrid: Akal.

Goetz, J. P. y LeCompte, M. D. (1988). Etnografía y diseño cualitativo en la investigación cualitativa. Madrid: Morata.

Hargreaves, D. J. (1996). The development of artistic and musical competence. En I. Deliège y J. A. Sloboda (Eds.), Musical beginings, (pp. 145-170). Oxford: Oxford University Press.

Hargreaves, D. J. (2002). Música y desarrollo psicológico. Barcelona: Graó.

Hernández, J. R., Hernández, J. A. y Milán, M. A. (2007). La creatividad asociada al talento musical en alumnos superdotados. Respuestas educativas. Ensayos. Revista de la Facultad de Educación de Albacete, 22, 83-98.

Jimeno, M. M. (2000). La investigación-acción en el contexto de la Educación Musical. Revista Electrónica de LEEME, 5, 1-6. Recuperado de https://ojs.uv.es/index.php/LEEME/ article/view/9694/9132

Kenny, D. T. (2008). Music Performance Anxiety. International Handbook of Musicians' Health and Wellbeing. Oxford, UK: Oxford University Press.

Kincheloe, J. L. (2012). Teachers as Researches. Qualitative Inquiry as a Path to Empowerment. Wiltshire: Routledge.

Kodály, Z. (1969). Pentatonic music. London: Boosey \& Hawkes.

Krumhansl, C. L. (2004). The Cognition of Tonality-as We Know it Today. Journal of New Music Research, 33(3), 253-268.

Krumhansl, C. L. y Kessler, E. (1982). Tracing the dynamic changes in perceived tonal organization in a spatial representation of musical keys. Psychological Review, 89, 334-368.

Laitz, S. G. (2012). The Complete Musician: An Integrated Approach to Tonal Theory, Analysis, and Listening. Oxford, UK: Oxford University Press.

LaRue, J. (1989). Análisis del estilo musical. Pautas sobre la contribución a la música del sonido, la armonía, la melodía, el ritmo y el crecimiento formal. Barcelona: Labor.

Lasuén, S. (2014). Alternativa a la enseñanza de la Armonía en los Conservatorios: hacia un enfoque conscientemente sincrónico. En Actas del Congreso CEIMUS III (Comp.) (pp. 345-357). Madrid: Enclave Creativa Ediciones.

Levitin, D. J. (2006). This is your brain on music: the science of human obsesión. New York, NY: Dutton Adult. 
Ley Orgánica 8/2013, de 9 de diciembre, para la Mejora de la Calidad Educativa (LOMCE).

Loebach, J. L., Conway, C. M. y Pisoni, D. B. (2009). Audition: Conginitive Influences. Encyclopedia of Perception. Recuperado de http://www.sageereference.com/perception/ Article_n50.html

López de Ārenosa, E. (2004). Apuntes sobre didáctica musical. Madrid. Enclave Creativa Ediciones.

López-Íñiguez, G., Pozo, J. I. y De Dios, M. J. (2014). The older, the wiser? Profiles of string instrument teachers with different experience according to their conceptions of teaching, learning, and evaluation. Psychology of Music, 42(2), 157-176.

Loui, P. y Wessel, D. (2007). Harmonic expectation and affect in Western music: Effects of attention and training. Perception and Psychophysics, 69(7), 1084-1092.

Mackamul, R. (1969). Lehrbuch der Gehörbildung. Band 1, elementare Gehörbildung. Kassel: Bärenreiter.

Malagarriga, T., Gómez, I. y Viladot, L. (2013). Bases de la propuesta. En T. Malagarriga y M. Martínez (Eds), Todo se puede expresar con música: los niños y niñas de 4 a 7 años piensan la música, hablan de música, crean música (pp. 17-78). Barcelona: DINSIC Publicacions Musicals.

Malbrán, S. (1996). Los atributos de la audición musical. Notas para su descripción. Eufonía. Didáctica de la Música, 2, 55-68.

Malbrán, S. (2007). El oído de la mente. Madrid: Akal.

Martenot, M. (1993). Principios fundamentales de formación musical y su aplicación. Madrid: Rialp.

Maykut, P. y Morehouse, R. (1994). Investigación cualitativa. Una guía práctica y filosófica. Barcelona: Hurtado.

McKernan, J. (1999). Investigación-acción y currículum. Métodos y recursos para profesionales reflexivos. Madrid: Ediciones Morata.

Miguel-Tobal, J. J. (1990). La ansiedad. En J. Mayor y L. Pinillos (Eds.), Tratado de Psicología General: Motivación y Emoción (Vol. 3), (pp. 309-344). Madrid: Alhambra.

Molina, E. (2008). La improvisación como sistema pedagógico. Funcionamiento, objetivos y resultados de la metodología IEM. En Actas del Congreso CEIMUS I (Comp.) (pp. 252272). Madrid: Enclave Creativa Ediciones.

Muñoz, E. y Bonastre, C. (2013). La expresividad en la música. Quodlibet. Revista de especialización musical, 52, 40-52.

Orff, C. y Keetman, G. (1950). Musik für Kinder. Mainz: Schott.

Patel, A. D. (2012). Language, music, and the brain: A resource-sharing framework. En P. Rebuschat, M. Rohrmeier, J. A. Hawkins \& I. Cross (Eds.), Language and music as cognitive systems (pp. 204-223). Oxford: Oxford University Press.

Pérez, J. (2011). La música a la vida quotidiana d'infants de dos anys. Anàlisi de situación musicals que es desenvolupen en context escolar. (Tesis doctoral). Univesitat Autònoma de Barcelona. Recuperado de http://www.tdx.cat/handle/10803/96096

Platt, J. R. y Racine, R. J. (1994). Detection of Implied Harmony Changes in Triadic Melodies. Music Perception, 11(3), 243-264.

Ponsatí, I. (2011). Avaluació d'una proposta didáctica per a la identificació auditiva dels intervals harmònics musicals. Una experimentació al primer curs dels ensenyaments especialitzats de grau profesional dels conservatoris de música de Catalunya. (Tesis doctoral). Universidad Autónoma de Barcelona. Recuperado de http://dialnet.unirioja.es/ servlet/tesis?codigo $=24235$ 
Puente-Ferreras, A. (2003). Memoria: cognición y aprendizaje. Fundamentos psicológicos. Madrid: Pirámide.

Rivas Navarro, M. (2008). Procesos cognitivos y aprendizaje significativo. Comunidad de Madrid. Consejería de Educación. Viceconsejería de Organización Educativa.

Roig-Francolí, M. A. (2011). Harmony in Context. New York, NY: McGraw-Hill.

Schafer, M. (1983). El compositor en el aula. Buenos Aires: Ricordi.

Schönberg, A. (1969). Structural functions of harmony. New York, NY: Norton \& Company. Sherman, R. R. y Webb, R. B. (1988). Qualitative research in education: A Focus. En R. R. Sherman y R. B. Webb (Eds.), Qualitative research in education: Focus methods (pp. 2-21). Filadelfia, PA: The Palmer Press.

Sierra, F. (2008). Constructivismo y Educación Auditiva. Música y Educación, 73, 110-116.

Sloboda, J. (1985). The Musical Mind. The Cognitive Psychology of Music. London: Oxford University Press.

Suzuki, S. (2007). Vivrec'estaimer. Marsella: Éditions Corroy.

Tafuri, J. (2006). ¿Se nace musical?: Cómo promover las aptitudes musicales de los niños. Barcelona: Grao.

Taylor, S. J. y Bodgan, R. (1986). Introducción a los métodos cualitativos de investigación. Buenos Aires: Paidós.

Torrado, J. A. y Pozo, J. I. (2006). Del dicho al hecho: de las concepciones sobre el aprendizaje a la práctica de la enseñanza de la música. En J. I. Pozo et al. (Eds), Nuevas formas de pensar la enseñanza y el aprendizaje: Las concepciones de profesores y alumnos (pp. 205-230). Barcelona: Graó.

Torres, M. J. (2011). La importancia de la educación auditiva, rítmica y vocal en la etapa de educación infantil. Revista Digital Innovación y Experiencias Educativas, 39. Recuperado de http://www.csi-csif.es/andalucia/modules/mod_ense/revista/pdf/ Numero_39/ MARIA_JOSE_TORRES_MENDEZ_02.pdf

Virues, R. A. (2005). Estudio sobre ansiedad. Revista Psicología Cientifica.com, 7(8). Recuperado de http://www.psicologia cientifica.com/ansiedad-estudio/

Ward, J. (1964). Método Ward. Pedagogía musical escolar. París: Desclée.

Willems, E. (1981). Bases psicológicas de la educación musical. Barcelona: Paidós.

\section{Fe de erratas}

Este artículo contiene varias erratas que pueden consultarse en:

Berrón Ruiz, E.; Monreal Guerrero, I.M.; Balsera Gómez, F.J. (2017). El conocimiento armónico como estrategia para mejorar la comprensión, la motivación y la creatividad en el aprendizaje del lenguaje musical, en Revista Electrónica Complutense de Investigación en Educación Musical,14, 305-327. Fe de erratas en: RECIEM. 2017, 14: 391-393. DOI: https://doi.org/10.5209/reci.74658 